University of Nebraska - Lincoln

DigitalCommons@University of Nebraska - Lincoln

$11-5-2004$

\title{
Disorder-induced depression of the Curie temperature in mechanically milled $\mathrm{GdAl}_{2}$
}

\author{
Marco Morales Torres \\ University of Nebraska - Lincoln, mmorales_torres2@unl.edu \\ D. S. Williams \\ University of Nebraska-Lincoln \\ P. M. Shand \\ University of Northern lowa \\ C. Stark \\ University of Northern lowa \\ T. M. Pekarek \\ University of North Florida, tpekarek@unf.edu
}

See next page for additional authors

Follow this and additional works at: https://digitalcommons.unl.edu/physicslesliepelecky

Part of the Physics Commons

Morales Torres, Marco; Williams, D. S.; Shand, P. M.; Stark, C.; Pekarek, T. M.; Yue, Lanping; Petkov, V.; and Leslie-Pelecky, Diandra, "Disorder-induced depression of the Curie temperature in mechanically milled GdAl2" (2004). Diandra Leslie-Pelecky Publications. 6.

https://digitalcommons.unl.edu/physicslesliepelecky/6

This Article is brought to you for free and open access by the Research Papers in Physics and Astronomy at DigitalCommons@University of Nebraska - Lincoln. It has been accepted for inclusion in Diandra Leslie-Pelecky Publications by an authorized administrator of DigitalCommons@University of Nebraska - Lincoln. 


\section{Authors}

Marco Morales Torres, D. S. Williams, P. M. Shand, C. Stark, T. M. Pekarek, Lanping Yue, V. Petkov, and Diandra Leslie-Pelecky 


\title{
Disorder-induced depression of the Curie temperature in mechanically milled $\mathbf{G d A l}_{2}$
}

\author{
M. A. Morales, ${ }^{1}$ D. S. Williams, ${ }^{1}$ P. M. Shand, ${ }^{2}$ C. Stark, ${ }^{2}$ T. M. Pekarek, ${ }^{3}$ L. P. Yue, ${ }^{1}$ V. Petkov, ${ }^{4}$ and D. L. Leslie-Pelecky ${ }^{1}$ \\ ${ }^{1}$ Department of Physics and Astronomy and Center for Materials Research and Analysis, University of Nebraska-Lincoln, \\ Lincoln, Nebraska 68588-0111, USA \\ ${ }^{2}$ Department of Physics, University of Northern Iowa, 205 Physics Building, Cedar Falls, Iowa 50614, USA \\ ${ }^{3}$ Department of Chemistry and Physics, University of North Florida, 4567 St. John's Bluff Road South, Jacksonville, Florida 32224, USA \\ ${ }^{4}$ Department of Physics, Central Michigan University, 203 Dow Science, Mt. Pleasant, Michigan 48859, USA
}

(Received 18 March 2004; revised manuscript received 14 May 2004; published 5 November 2004)

\begin{abstract}
The effect of disorder on the ferromagnetic transition is investigated in mechanically milled $\mathrm{GdAl}_{2} \cdot \mathrm{GdAl}_{2}$ is a ferromagnet when crystalline and a spin glass when amorphous. Mechanical milling progressively disorders the alloy, allowing observation of the change from ferromagnetic to a disordered magnetic state. X-ray diffraction and pair-distribution-function analysis are used to determine the grain size, lattice parameter, and mean-squared atomic displacements. The magnetization as a function of temperature is described by a Gaussian distribution of Curie temperatures. The mean Curie temperature decreases with decreasing lattice parameter, where lattice parameter serves as a measure of defect concentration. Two different rates of change with lattice parameter are observed: the first, slower rate occurs while grain size is changing, and the grain size is constant in the second regime. The breadth of the Curie temperature distribution is linear with lattice parameter. Milled and annealed $\mathrm{GdAl}_{2}$ shows that the mean Curie temperature has the same general dependence on lattice parameter as milled, but unannealed $\mathrm{GdAl}_{2}$, while the breadth of the Curie temperature distribution is different in annealed samples compared to the unannealed materials.
\end{abstract}

DOI: 10.1103/PhysRevB.70.184407

PACS number(s): 75.75.+a, 75.50.Tt

\section{INTRODUCTION}

Understanding how different types of disorder affect ferromagnetic transitions is a fundamental and important problem in magnetism. Although the magnetic properties of many nanostructured materials can be explained by length-scale effects (such as grain or particle size), atomic-level disorder also can have a profound effect on magnetic properties. For example, Gd films ${ }^{1-3}$ grown under different conditions (and thus with different levels of structural disorder) can have significantly different Curie temperatures. When the disorder becomes large enough, the ferromagnetic phase can become magnetically glassy.

$\mathrm{GdAl}_{2}$ is an ideal system for investigating the effects of disorder on the paramagnetic-ferromagnetic (PM-FM) transition. Despite complex magnetic behavior, much of the magnetic behavior of the Laves $R \mathrm{Al}_{2}$ (where $R$ is a rare earth) compounds can be explained by a Rudermann-KittelKasuya-Yoshida (RKKY) model of indirect exchange. Two different types of disorder must be considered: structural (the deviation of an atom from its expected position) and chemical (whether an atom is on the correct sublattice). Crystalline $\mathrm{GdAl}_{2}$ is a ferromagnet with the $\mathrm{MgCu}_{2}$ fcc structure (lattice parameter $a_{0}=0.7899 \mathrm{~nm}$ ), a Curie temperature $T_{C}$ of $170 \mathrm{~K}$, and a small magnetocrystalline anisotropy. ${ }^{4,5}$ Amorphous $\mathrm{GdAl}_{2}$ exhibits classic spin-glass behavior-a peak in the zero-field-cooled (ZFC) susceptibility at peak temperature $T_{p}=60 \mathrm{~K}$, irreversibility between the ZFC and field-cooled (FC) magnetizations, ${ }^{6}$ and critical scaling of ac susceptibility measurements. ${ }^{7}$ Mechanically milling initially crystalline $\mathrm{GdAl}_{2}$ produces a spin-glass-like peak, irreversibility between FC and ZFC magnetizations, and a decrease in the magnitude of the ferromagnetic transition. ${ }^{8-11}$ The spinglass-like peak in $M(T)$, however, occurs $20-40 \mathrm{~K}$ higher than that in amorphous $\mathrm{GdAl}_{2}$. Zhou and Bakker interpret the low-temperature peak as evidence of a "new class" of spin glass in which milling-induced disorder creates the necessary randomness and frustration, ${ }^{8,9}$ although there remains disagreement on whether the peak is due to a true spin-glass transition. ${ }^{12,13}$ The progressive disordering of $\mathrm{GdAl}_{2}$ by mechanical milling thus allows us to track how the paramagnetic-ferromagnetic transition changes as the crystalline order is destroyed and how disorder affects the nature of the ferromagnetic phase.

\section{SAMPLE FABRICATION}

The versatility of mechanical milling makes it a valuable tool for altering a material's structure in a controlled manner. In contrast to mechanical alloying, in which two or more separate components are combined into a single homogeneous material, mechanical milling transforms an initially crystalline material by introducing defect structures (dislocations and vacancies) and atomic-scale chemical disorder.

Gadolinium chips (99.9\% pure) and aluminum pellets (99.99\% pure) were arc melted in an argon atmosphere in the proper proportions to produce $\mathrm{GdAl}_{2}$. The resulting buttons were turned and the procedure repeated several times to ensure homogeneous mixing. The alloy was crushed, milled for two hours in a tungsten-carbide vial under an argon atmosphere to produce a fine powder, and then annealed for $24 \mathrm{~h}$ at $800{ }^{\circ} \mathrm{C}$ under vacuum to remove milling-induced stress. $\mathrm{X}$-ray diffraction and energy-dispersive $\mathrm{x}$-ray measurements confirm that the powders produced by this process are crystalline and highly ordered, with no tungsten carbide or oxide contamination. This material is referred to as the " $0 \mathrm{~h}$ " or "unmilled" sample. 


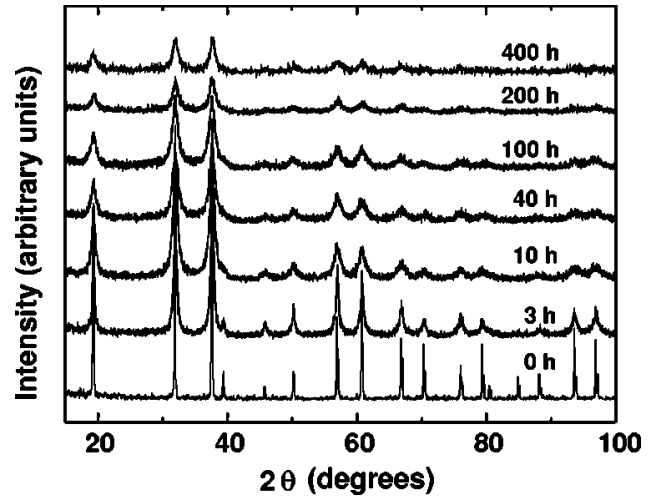

FIG. 1. X-ray diffraction patterns showing the decrease in peak height and peak broadening as the sample is milled.

Two milling techniques were used. In low-intensity milling, the initially crystalline powders were milled in a Fritsch

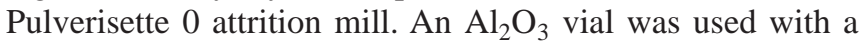
ball-to-powder mass ratio of $\sim 21: 1$. Samples were milled for times up to $2500 \mathrm{~h}$, stopping only to remove small amounts of powder for measurements. High-intensity milling was performed in a SPEX 8000 mill with ball-to-powder mass ratios ranging from $0.6: 1$ to $3: 1$. 15-min milling periods were alternated with 15 -min rest periods to reduce heating, and the vial was turned every $2 \mathrm{~h}$ to reduce clumping. Small amounts of powder were removed periodically for structural and magnetic measurements. All sample handing was performed in an Ar atmosphere to prevent oxidation.

\section{STRUCTURAL CHARACTERIZATION}

The complex dependence of the final structure on ball-topowder mass ratio, milling/resting times, internal temperature, and other variables means that milling time is not a robust enough parameter to use for comparison of samples from different labs, or even from different runs. Comparing data within a single run, however, is useful for a qualitative overview of the disordering process.

Figure 1 shows $\mathrm{x}$-ray diffraction patterns as functions of milling time for a series of samples produced by highintensity milling. As is the case for all milling runs, peak intensity decreases and peak width broadens with milling time, with the largest changes occurring during the first $10-20 \mathrm{~h}$ of milling. The grain size decreases rapidly from its initial value (which is on the order of microns) and reaches a terminal grain size of $\sim 8 \mathrm{~nm}$ after about $40 \mathrm{~h}$ of highintensity milling. The process is similar with low-intensity milling, but occurs at a slower rate. The grain size decreases to $\sim 15 \mathrm{~nm}$ after $2500 \mathrm{~h}$ of low-intensity milling.

Measurements were made at the Advanced Photon Source, Argonne National Laboratory to further characterize the structure. Samples-either thin $(\sim 0.5 \mathrm{~mm})$ plates of powder between Kapton tapes or powder sealed in glass capillary tubes-were measured at beamlines 1-ID (at an x-ray energy of $80 \mathrm{keV}$ ) or 11-ID (with an x-ray energy of $115 \mathrm{keV})$. Data were corrected for instrumental effects and normalized to absolute (electron) units. ${ }^{14}$ Bragg diffraction

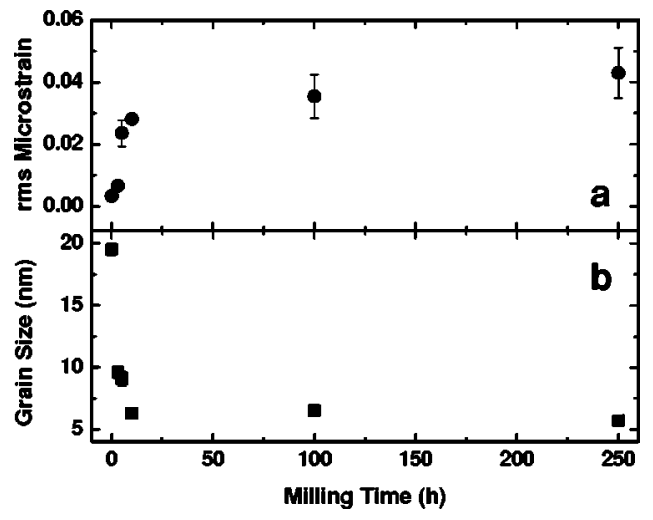

FIG. 2. (a) The root-mean-square microstrain and (b) the grain size as functions of milling time.

peaks were analyzed to determine the rms microstrain and grain size, which are shown in Fig. 2 as functions of milling time. If only grain-size effects are considered in analyzing $\mathrm{x}$-ray diffraction data, different diffraction peaks produce different values for the grain size; however, an analysis that includes grain size and strain ${ }^{15,16}$ produces a consistent mean grain size. Figure 2 shows quantitatively that the largest changes in the grain size and strain in high-energy-milled samples occur during the first hours of milling.

Figure 3 shows the reduced structure function $Q[S(Q)$ -1] for unmilled $\mathrm{GdAl}_{2}$, and $\mathrm{GdAl}_{2}$ milled for $100 \mathrm{~h}$ and $400 \mathrm{~h}$. The decrease in peak intensity with milling and the reduction in the value of $Q$ to which peaks are observed are indicative of the disordering process. The experimental pair distribution functions were fit using PDFFIT ${ }^{17}$ to obtain the unit-cell constant $a$ and mean-squared atomic displacements $u_{\mathrm{Gd}}$ and $u_{\mathrm{Al}}$. Figure 4 shows the lattice parameter and meansquared atomic displacements versus milling time for a single milling run. As in all milling runs, $a$ decreases and $u_{\mathrm{Gd}}$

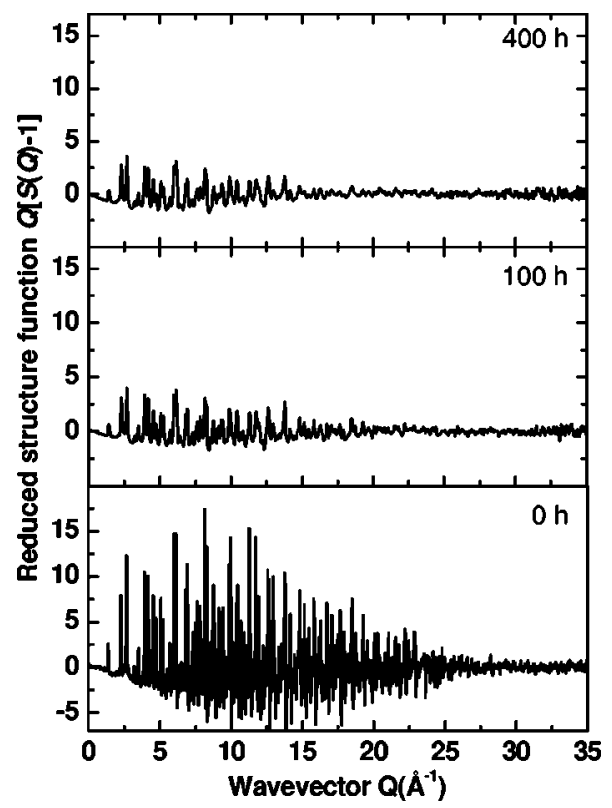

FIG. 3. Reduced structure functions for unmilled $\mathrm{GdAl}_{2}$ (bottom) and $\mathrm{GdAl}_{2}$ milled for times of $100 \mathrm{~h}$ (middle) and $400 \mathrm{~h}$ (top). 


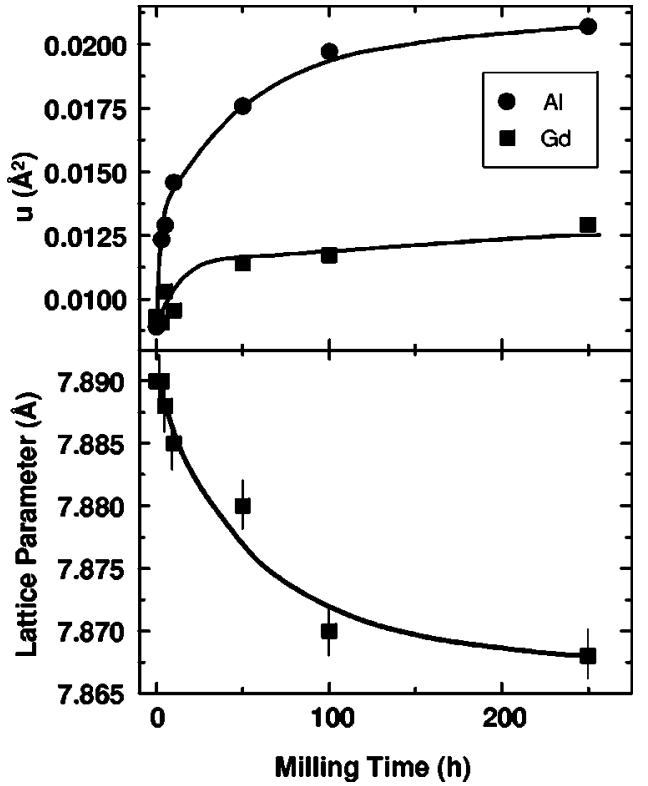

FIG. 4. (a) Mean-squared atomic displacement and (b) lattice parameter as functions of milling time. The lines are guides to the eye.

and $u_{\mathrm{Al}}$ increase with milling. The value of $u_{\mathrm{Gd}}$ remains smaller than $u_{\mathrm{Al}}$ throughout the entire range of disorder.

\section{MAGNETIC MEASUREMENTS}

Samples for magnetic measurements were prepared in an Ar atmosphere and sealed in paraffin-filled polyethylene bags to prevent oxidation and rotation of the particles during measurement. Measurements were made in a SQUID magnetometer and a Lakeshore ac susceptometer as functions of field and temperature.

Figure 5(a) shows the ZFC magnetization as a function of temperature for $\mathrm{GdAl}_{2}$ milled for times from $0 \mathrm{~h}$ to $44 \mathrm{~h}$. An expanded view of the transition region is shown in Fig. 5 (b). Although a broad peak is observed near $40-60 \mathrm{~K}$, this paper will focus on the changes in the paramagneticferromagnetic transition that are highlighted in Fig. 5(b).

\section{A. The paramagnetic regime}

Crystalline and amorphous $\mathrm{GdAl}_{2}$ obey a Curie-Weiss behavior above their respective transition temperatures. ${ }^{18}$ Our unmilled $\mathrm{GdAl}_{2}$ has an effective moment of $(8.60 \pm 0.04) \mu_{\mathrm{B}}$ and a Curie-Weiss temperature of $171 \pm 1 \mathrm{~K}$. Conductionelectron enhancement effects are responsible for the moment being higher than the value of $7.9 \mu_{\mathrm{B}}$ expected from $\mathrm{Gd}^{3+}$ ions. ${ }^{19}$ We have demonstrated previously ${ }^{11}$ that milling transforms the sample from a single phase that can be fitted by a Curie-Weiss form to a combination of phases that requires a Curie-Weiss and a Curie form,

$$
\chi(T)=\frac{c_{1}}{T-\theta}+\frac{c_{2}}{T},
$$

where the first term describes the FM phase and the second the phase generated by milling. As milling time increases, $c_{1}$

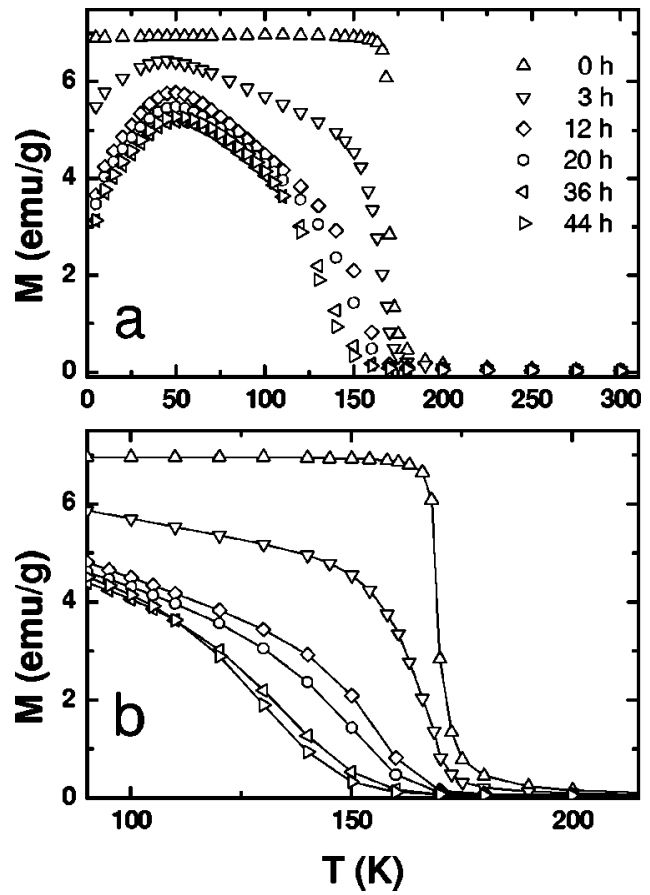

FIG. 5. $M_{Z F C}(T)$. The lower figure shows details of the behavior near $T_{C}$.

decreases and $c_{2}$ increases, with the sum $c_{1}+c_{2}$ remaining approximately constant. The enhancement of the second phase at the expense of the first is consistent with the decrease in magnitude of the FM transition relative to the lowtemperature peak. That the second phase can be described by a pure Curie form suggests a significant reduction in the average exchange strength in the second phase relative to the first at these temperatures. The fraction of the second component increases with milling time, so the reduction in exchange is at least partially due to milling-induced disorder.

\section{B. The PM-FM transition}

Figure $6(d M / d T$ vs $T)$ emphasizes the shift and broadening of the PM-FM transition with increasing disorder. The

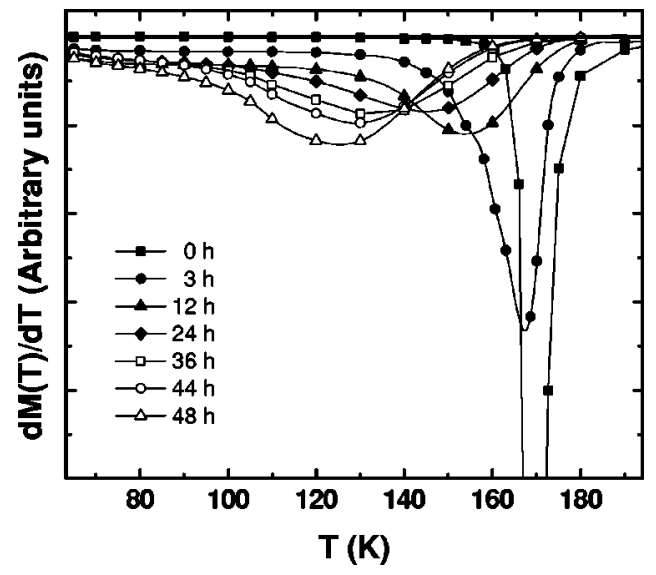

FIG. 6. $d M_{Z F C}(T) / d T$ for various milling times as a function of temperature. All measurements were made at a field of 100 Oe. 


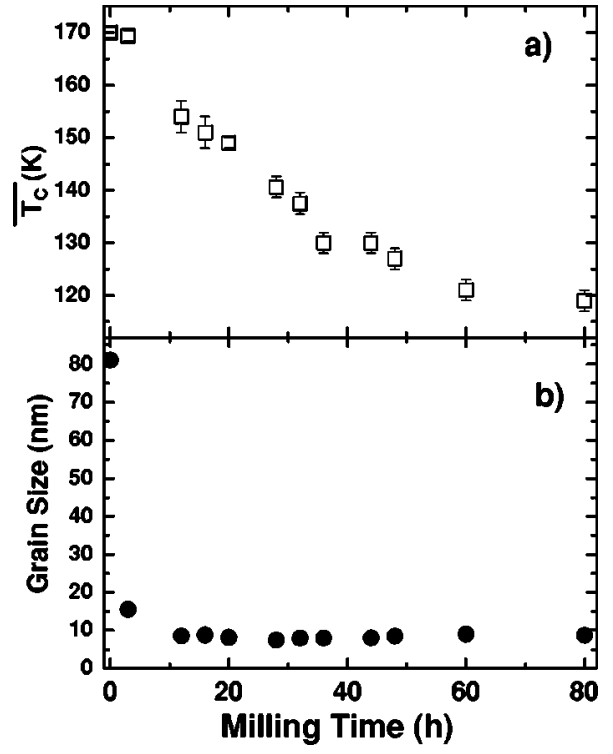

FIG. 7. Changes in (a) $\bar{T}_{C}$ and (b) grain size with milling time in mechanically milled $\mathrm{GdAl}_{2}$.

broadening is attributed to the distribution of local environments created by disorder. $T_{C}$ cannot be determined accurately from the minimum of $d M(T) / d T$, and instead is found by fitting $M(T)$ to a distribution of Curie temperatures: ${ }^{20-22}$

$$
M(T)=m_{0} \int_{T_{C}}\left(\frac{T_{C}-T}{T_{C}}\right)^{\beta} \theta\left(T_{C}-T\right) \rho\left(T_{C}\right) d T_{C},
$$

where $\rho$ is a Gaussian with breadth $\Delta T_{C}$ and mean value $\bar{T}_{C}$, and $\theta$ is the Heaviside step function. $\bar{T}_{C}$ obtained from Eq. (2) is larger than that found from the derivative method, with the difference between the values increasing with increasing disorder and increasing measuring field. ${ }^{20} \bar{T}_{C}$ is essentially independent of the measuring field; however, $\Delta T_{C}$ depends on measuring field as $H^{1 / \eta}$ where $\eta \sim 1-1.5$ for $\mathrm{GdAl}_{2}{ }^{20}$

Two effects shown in Fig. 5 must be explained: the shift in $\bar{T}_{C}$ and the broadening of the transition with milling time. A possible explanation for the decrease of $\bar{T}_{C}$ with milling time is finite-size effects due to decreasing grain size. Figure 7 ( $\bar{T}_{C}$ and grain size versus milling time) shows that the most significant changes in $\bar{T}_{C}$ occur in the region where grain size has become constant, indicating that the depression is not a grain-size effect.

The introduction of vacancies and antisite defects by milling produces a decrease in the lattice parameter (as shown in Fig. 4). The lattice parameter change $\Delta a$ is the difference between the lattice parameter $a$ and the bulk lattice parameter $a_{0}\left(\Delta a=a_{0}-a\right)$. The lattice parameter of mechanically milled $\mathrm{GdAl}_{2}$ is always smaller than the bulk value, so $\Delta a$ is positive. $\mathrm{GdAl}_{2}$ disorders by a quadruple-defect process. ${ }^{23,24}$ The size difference between $\mathrm{Gd}$ and $\mathrm{Al}$ allows an $\mathrm{Al}$ atom to substitute on the Gd sublattice, but not vice versa. The overall $\mathrm{MgCu}_{2}$ structure is retained because $\mathrm{Gd}$ atoms are present

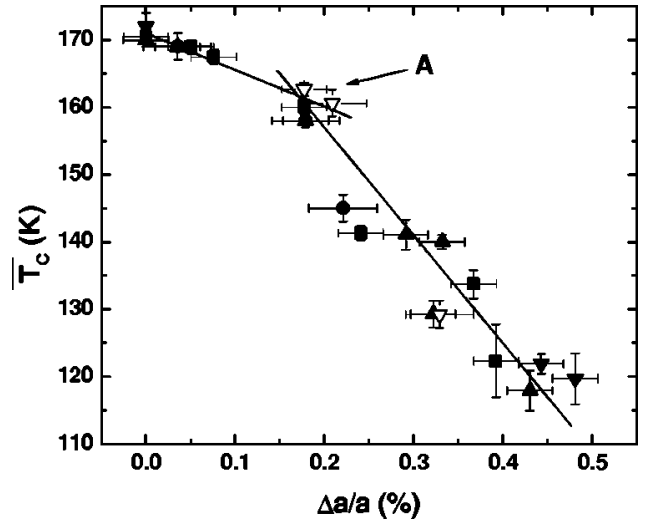

FIG. 8. $\bar{T}_{C}$ as a function of percent change of lattice parameter. Different symbols represent samples from different milling runs. The open symbols represent samples milled and subsequently annealed. The letter A refers to a group of points with similar values of $T_{C}$ and $a$, but very different grain sizes.

as defects or on their own sublattice. ${ }^{25}$ Preliminary extended $\mathrm{x}$-ray absorption fine-structure (EXAFS) measurements confirm that the primary disorder is in the Gd sublattice, producing a decrease in the mean Gd coordination number and an overall decrease in the lattice parameter.

$\mathrm{GdAl}_{2}$ exhibits striking differences from other $\mathrm{Gd} X_{2}$ alloys that have been studied in their nanostructured form. In $\mathrm{GdPt}_{2}$, and $\mathrm{GdIr}_{2}, T_{C}$ increases as the lattice parameter decreases. ${ }^{26}$ The difference in the direction of the $\bar{T}_{c}$ change in $\mathrm{GdAl}_{2}$ is attributed to the $p$ nature of Al. NMR measurements indicate that the atomic character of the conduction electrons is mainly $5 d$-like for $\mathrm{GdAl}_{2}$ and $6 s$-like for $\mathrm{GdPt}_{2}$ and $\mathrm{GdRh}_{2} \cdot{ }^{27-29}$ Modder et al. suggest that decreasing the Gd-Gd distance should produce a decrease in $T_{C}{ }^{26}$

In contrast, however, are data from experiments using pressure to decrease the lattice parameter of bulk $\mathrm{GdAl}_{2} \cdot{ }^{30,31}$ $T_{C}$ increases with decreasing lattice parameter at a rate of $d T_{C} / d p=+7.1 \mathrm{~K} / \mathrm{GPa}$. The change in $T_{C}$ is significantly smaller (from roughly $165 \mathrm{~K}$ at $0.1 \mathrm{GPa}$ to $167 \mathrm{~K}$ at $333 \mathrm{GPa}$ ) than the magnitude of the change observed in nanostructured $\mathrm{GdAl}_{2}$.

Figure 8 shows $\bar{T}_{C}$ obtained from fitting to Eq. (2) as a function of the fractional change in lattice parameter. All measurements were made in a field of $100 \mathrm{Oe}$, with the exception of the solid triangles, which were made in a field of 200 Oe. There is no significant difference between values of $\bar{T}_{C}$ obtained at these two fields. ${ }^{20}$ The critical exponent $\beta$ can vary between 0.3 and 0.6 with almost no change in $\bar{T}_{C}$. The error bars indicate the range of $\bar{T}_{C}$ for $\beta$ from 0.3 to 0.6 .

Figure 9 shows the breadth of the distribution $\Delta T_{C}$ from Eq. (2) as a function of $\Delta a / a$. Since $\Delta T_{C}$ can change from 2 to $4 \mathrm{~K}$ for measurements made at $100 \mathrm{Oe}$ versus those made at $200 \mathrm{Oe}$, only measurements made at $100 \mathrm{Oe}$ are shown in Fig. 9. Excluding the open symbols, which represent data from annealed samples, the dependence of $\Delta T_{C}$ on percent change of lattice parameter is roughly linear with a slope of $34 \mathrm{~K} / \%$ and an intercept of $2.9 \mathrm{~K}$. The nonzero 


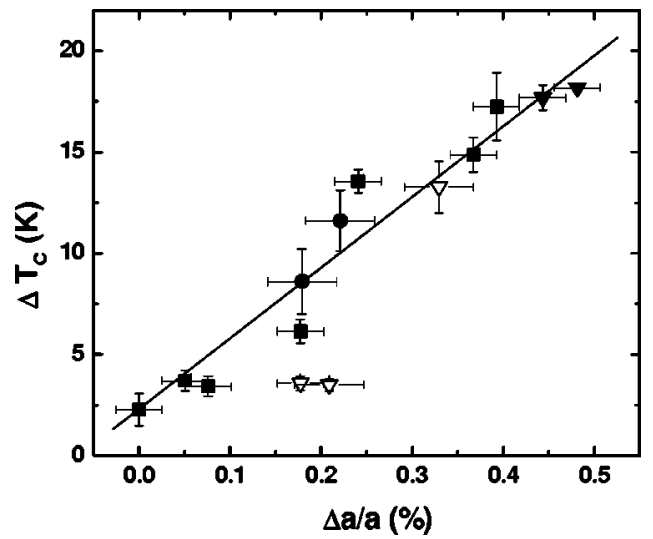

FIG. 9. The dependence of the distribution breadth $\Delta T_{C}$ on percent change in lattice parameter in mechanically milled $\mathrm{GdAl}_{2}$. Different symbols represent samples from different milling runs. Open symbols represent samples that were milled and subsequently annealed.

intercept is likely due to field broadening, which is nonnegligible at $100 \mathrm{Oe}^{20}$

ac magnetic susceptibility measurements were made to investigate how disorder affects the time-dependent magnetic response. Figure 10 shows a slight frequency dependence in the real part of the ac susceptibility $\chi^{\prime}$ and a more noticeable frequency dependence in the imaginary part of the ac susceptibility $\chi^{\prime \prime}$. Figure 11 shows that a dc bias field shifts the PM-FM transition to significantly lower temperatures, as indicated by the position of the shoulder in $\chi^{\prime}$ and the peak in $\chi^{\prime \prime}$.

Levin et al. ${ }^{5}$ observed a negative $\chi^{\prime \prime}$ in bulk $\mathrm{GdAl}_{2}$, which we do not observe in mechanically milled $\mathrm{GdAl}_{2}$. They attribute the negative $\chi^{\prime \prime}$ to domain-wall oscillations, which

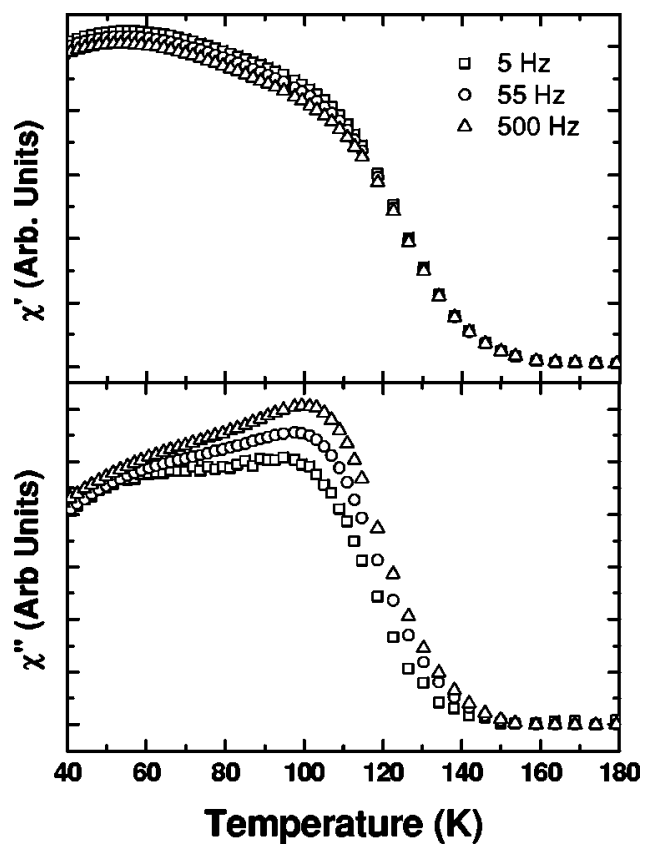

FIG. 10. The real (top) and imaginary (bottom) susceptibilities of a sample milled for $350 \mathrm{~h}$ measured at 1 Oe with no dc bias field.

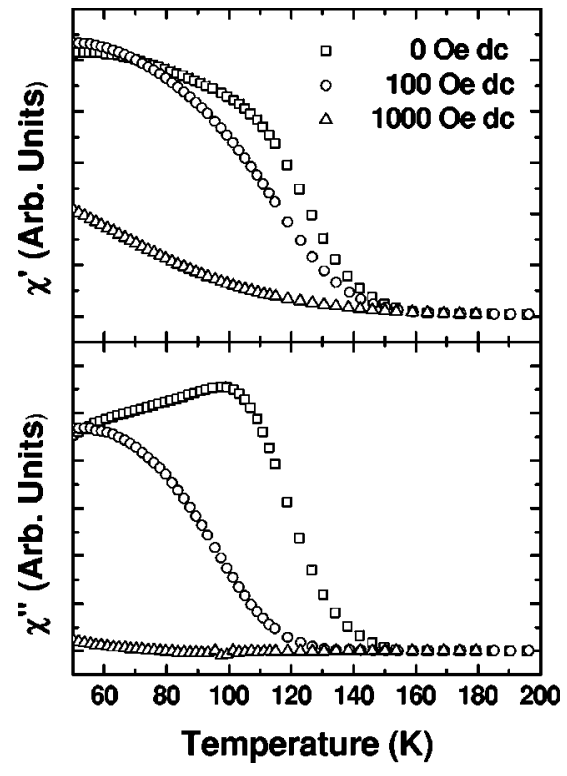

FIG. 11. The dependence of the real (top) and imaginary (bottom) susceptibility as a function of temperature and dc bias field measured at $55 \mathrm{~Hz}$ for a sample milled for $350 \mathrm{~h}$.

can occur in soft ferromagnets and even in stronger ferromagnets close to $T_{C}$ where the coercivity has not become appreciable. We have shown previously that disordered $\mathrm{GdAl}_{2}$ exhibits a $50-70$ Oe coercivity even above $T_{C}$, which may explain the difference in sign. ${ }^{20}$

\section{Mean lattice parameter versus disorder}

Annealing produces grain growth and atomic-level reordering, depending on the annealing conditions. All of the samples shown in Fig. 12 were initially milled for $590 \mathrm{~h}$. $M / H$ vs $T$ is shown in Fig. 12 for unannealed $\mathrm{GdAl}_{2}, \mathrm{GdAl}_{2}$ annealed at 400 and $600{ }^{\circ} \mathrm{C}$ for $10 \mathrm{~min}$, and annealed at $800{ }^{\circ} \mathrm{C}$ for $48 \mathrm{~h}$. Measurements were made in a field of 100 Oe.

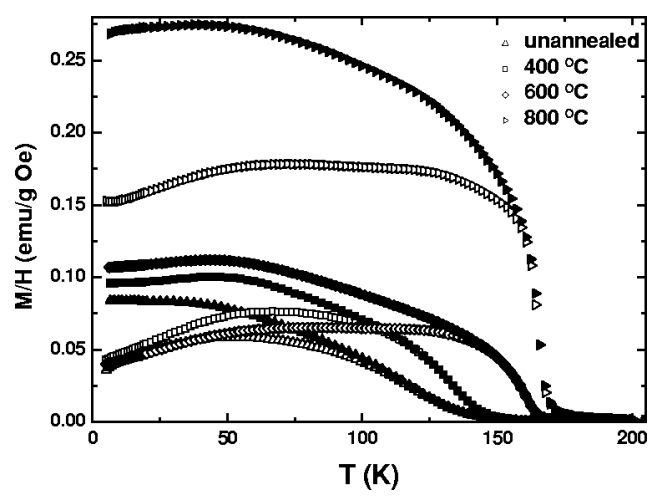

FIG. 12. $\chi(T)$ for $\mathrm{GdAl}_{2}$ milled for $590 \mathrm{~h}$ (triangles), then annealed $400{ }^{\circ} \mathrm{C}$ (squares), $600{ }^{\circ} \mathrm{C}$, (diamonds), and $800{ }^{\circ} \mathrm{C}$ (rightpointing triangles). All annealing times were for $10 \mathrm{~min}$ with the exception of the $800{ }^{\circ} \mathrm{C}$ anneal, which was for $48 \mathrm{~h}$. Open symbols represent ZFC and closed symbols FC measurements. 
Regardless of annealing conditions, the high-temperature data require both a Curie-Weiss and a Curie function [Eq. (1)] for a satisfactory fit. The Curie parameter $c_{1}$ (corresponding to the ferromagnetic phase) increases with increasing annealing temperature while $c_{2}$ decreases with increasing annealing temperature, and the Curie-Weiss temperature $\theta$ increases with increasing annealing temperature.

The most interesting aspect of the annealed samples is how their magnetic behavior compares with unannealed samples, especially in the case where lattice parameters are comparable. The annealed samples (represented by open symbols in Figs. 8 and 9) follow the same general relationship between $\bar{T}_{C}$ and $\Delta a / a$ as found for the milled samples. The lack of a grain-size effect is emphasized by the cluster marked $A$ in Fig. 8, which contains samples having roughly the same $\bar{T}_{C}$ and lattice parameter but with grain sizes ranging from 8 to $18 \mathrm{~nm}$.

In contrast, Fig. 9 shows that the breadths of annealed samples do not exhibit the same dependence on lattice parameter as their unannealed counterparts. Annealing markedly decreases the transition breadth. Both $u_{\mathrm{Gd}}$ and $u_{\mathrm{Al}}$ increase with increasing milling time. Annealing increases $u_{\mathrm{Gd}}$, but decreases $u_{\mathrm{Al}}$, indicating that $\mathrm{Al}$ atoms move closer to their equilibrium crystalline positions while $\mathrm{Gd}$ atoms exhibit overall increased disorder after this annealing. For a 500-h milled sample annealed at $600{ }^{\circ} \mathrm{C}$ for $10 \mathrm{~min}$, $u_{\mathrm{Gd}}$ increases from $0.0110( \pm 0.0005) \AA^{2}$ to 0.0190 $( \pm 0.0005) \AA^{2}$, while $u_{\mathrm{Al}}$ decreases from 0.0240 $( \pm 0.0005) \AA^{2}$ to $0.0070( \pm 0.0005) \AA^{2}$. The starting crystalline $\mathrm{GdAl}_{2}$ has a value $u_{\mathrm{Al}}=0.0080( \pm 0.0005) \AA^{2}$.

The overall form of $M(T)$ in annealed samples is significantly different from that of samples having the same $\bar{T}_{C}$, but made by milling with no annealing (e.g., compare Fig. 12 with Fig. 5). Samples annealed at $T>400{ }^{\circ} \mathrm{C}$ show a broad plateau between the two transitions that is not evident in unannealed samples.

\section{DISCUSSION}

A true PM-FM phase transition requires the correlation length to diverge across the entire sample. Defects restrict the divergence of the correlation length at $T_{C}$ in disordered materials, allowing FM order over only restricted length scales. ${ }^{32}$ Those length scales, however, need not correspond to a grain size. The strong exchange interactions between spins in a FM means that once a region exceeds $\sim 1 \mathrm{~nm}$, ordered spins behave as they would if the correlation length had diverged in the usual sense; however, different parts of the sample order at different temperatures. ${ }^{33}$ This picture is reinforced by neutron scattering measurements in chemically disordered (but single crystal) reentrant spin glass $\mathrm{Fe}_{0.7} \mathrm{Al}_{0.3}$. These measurements show that the dynamics below $T_{C}$ (but above the low-temperature peak) are qualitatively different from those of a conventional FM. ${ }^{32}$ Although there is overall FM order, substantial disorder at finite lengths produces remnant short-range spin correlations. The FM state changes from long-range order to short-range order with increasing disorder. The introduction of disorder in mechanically milled $\mathrm{GdAl}_{2}$ should have similar effects.
Since the disordering process occurs randomly, different regions of the sample will have different local environments. In particular, ejected $\mathrm{Gd}$ atoms are likely to segregate at grain boundaries, forming Gd-rich grain boundary regions and $\mathrm{Gd}$-poor $\mathrm{GdAl}_{2}$ grains. Annealing a sample milled for $590 \mathrm{~h}$ at $800^{\circ} \mathrm{C}$ for $48 \mathrm{~h}$ (a similar annealing condition as that used to create the starting material) does not reorder the sample back into the original $\mathrm{GdAl}_{2}$ structure, but instead produces $\mathrm{GdAl}_{2}$ and $\mathrm{Gd}_{2} \mathrm{Al}$ phases. The Gd-rich and $\mathrm{Gd}$ poor regions likely respond differently to annealing, with the Gd-poor $\mathrm{GdAl}_{2}$ phase retaining a smaller lattice parameter than bulk due to the Gd vacancies.

The dependence of $T_{C}$ on lattice parameter is not a volume effect in the traditional sense, as the change in the lattice parameter does not reflect an overall volume change of the unit cell, but the introduction of vacancies and antisite defects. Figure 8 shows that the decrease of $\bar{T}_{c}$ with decreasing lattice parameter can be separated into two regimes with different rates. The dividing line corresponds approximately to the point at which grain size reaches a constant value. (Note that differences in milling dynamics between runs means that the terminal grain size can be reached after different milling times in different runs.) The $\bar{T}_{c}$ depression observed by Zhou's group ${ }^{8,9,26}$ for $\Delta a / a<0.2 \%$ is consistent with our observations: their samples with similar lattice parameters have $T_{C}$ 's comparable to ours, despite having different grain sizes. Their samples, however, either did not show a ferromagnetic transition for $\Delta a / a>$ about $0.2 \%$, or the transition was so broad as to preclude reliable identification of $T_{C}$.

It is likely that the changes in the first regime include effects due to grain refinement and point defects, while the second regime only has contributions from point defects and the different nature of the defects is responsible for the different slopes. Low-intensity milling may allow an extended study of the first regime. The interaction between $\mathrm{Gd}$ atoms in $\mathrm{GdAl}_{2}$ is $\mathrm{RKKY}{ }^{31}$ mediated by $5 d$ electrons:

$$
T_{C}=-\frac{3 \pi}{4 k_{B}} \frac{Z^{2} \Gamma^{2}}{E_{F}} G \sum_{i} F\left(2 k_{F} r_{i}\right) e^{-r_{i} / \lambda}
$$

where $Z$ is the number of conduction electrons per atom, $r_{i}$ is the distance between the reference atoms and other atoms, $\Gamma$ is the exchange integral, $G$ is the de Gennes factor, $F$ is an oscillating function that describes the RKKY interaction, $\lambda$ is the mean free path, and the sum is taken over all $\mathrm{Gd}$ atoms (excluding the reference site). ${ }^{34} \mathrm{~A}$ uniform change in volume does not affect the sum over the function $F$ or $Z$, but does change the electronic band structure and thus leads to the increase in $T_{C}$ as observed experimentally in bulk $\mathrm{GdAl}_{2} \cdot{ }^{30,31}$

The milling-induced disorder randomizes the distances between atoms $r_{i}$, and introduces vacancies. In the freeelectron approximation, $k_{F}$ is proportional to $n^{1 / 3}$, where $n$ is the free-electron density. Spatial variations in $n$ (and thus in $k_{F}$ ) will affect the periodicity of the terms in the summation of Eq. (3). Added to this effect is the random displacement of $r_{i}$ from the lattice points, which further increases the 
incoherence of the terms in the summation with respect to each other. Vacancies will decrease the number of terms in the summation. The effect of disorder is thus to decrease $T_{C}$ and spatial variations in disorder produce a range of $T_{C}$ values that broaden the transition. Theoretical calculations of the electronic structure in $\operatorname{Gd}\left(\mathrm{Al}_{1-x} M_{x}\right)$ shows that increasing the dopant concentration for nonmagnetic $M$ decreases the density of states at the Fermi level and thus predicts a lower $T_{C}$ in the presence of substitutional disorder. ${ }^{35} T_{C}$ decreases regardless of whether the number of conduction electrons per unit cell increases or decreases and the observed decreases in $T_{C}$ can be up to $50 \%$, depending on $M$ and the concentration. The mean free path of the conduction electrons decreases when disorder is introduced. Apart from diminishing the strength of the interaction, the effect of the exponential damping is to decrease the relative importance of fartherneighbor interactions. The magnetic behavior increasingly should be dominated by the local environment. One caveat is that the free-electron RKKY theory is likely to be less valid for non- $6 s$ conduction electrons, and the specific role of the $5 d$ electrons in determining $T_{C}$ is less clear.

The breadth of the transitions suggests that cooperative effects among grains are small and intergrain exchange coupling is relatively weak at these temperatures, although intergrain interactions may become considerable at lower temperatures. A true PM-FM transition should not exhibit frequency dependence in $\chi^{\prime}$ or $\chi^{\prime \prime}$ at $T_{C} ; 36,37$ however, the mechanically milled samples do show frequency dependence (weak in $\chi^{\prime}$ and stronger in $\chi^{\prime \prime}$, as shown in Fig. 10). While grain size does not appear to determine $\bar{T}_{C}$, the dynamics likely are affected by the range of FM order. The increasing $\mathrm{dc}$ bias field orients spins in the direction of the field (essentially saturating the grains) and suppresses the response to the small ac field. As measurements are made in an increasingly larger bias field, the PM-FM transition in $\chi^{\prime}$ and $\chi^{\prime \prime}$ almost disappears entirely, as seen in Fig. 11.

The choice of a Gaussian distribution to model the $T_{C}$ distribution could be questioned. Although the distribution of grain sizes should be log-normal, the lattice parameter change is not directly linked to grain size, so a log-normal distribution is not an obvious default choice. The types of defects introduced by milling favor decreasing the lattice parameter within the grains, which might suggest using a nonsymmetric distribution that reflects the asymmetry of the lattice getting smaller and not larger. On the other hand, surface anisotropy can be significant in smaller grains, and this would tend to increase the Curie temperature. Regardless of the functional form used to model the breadth, the raw data clearly show that the annealed samples have a significantly different dependence of their breadth on the lattice parameter, despite having the same dependence of $\bar{T}_{c}$ on lattice parameter.

The behavior of the breadth of the PM-FM transition offers an additional way to investigate the character of the transition as a function of disorder, especially given the very different behavior of milled and milled/annealed samples having the same $\bar{T}_{C}$, but breadths that differ by up to $20 \mathrm{~K}$. Substantive differences in the shapes of $M(T)$ in samples with the same $\bar{T}_{c}$ and lattice parameter must be investigated to understand the nature of the disordered phase. The PM-FM transition never disappears entirely in our materials as was seen by Zhou and Bakker. In contrast, their $M(T)$ curves show a very long tail at high temperatures, whereas ours have much steeper slopes. This emphasizes the necessity of atomic-level characterization using a technique such as EXAFS to probe the details of the local environments and correlating these structural details to the magnetic behavior.

\section{CONCLUSIONS}

$\mathrm{GdAl}_{2}$ is an ideal system for studying how chemical and structural disorder affect the magnetic properties of ferromagnets. We have shown that modeling the ferromagneticparamagnetic transition is best accomplished by fitting $M(T)$ to a distribution of Curie temperatures, with the mean Curie temperature $\bar{T}_{C}$ correlated to the lattice parameter, and the distribution breadth $\Delta T_{C}$ reflecting the disorder of the local environment. These two parameters provide a more accurate description of the system than from finding $T_{C}$ from the minimum of $d M(T) / d T$ and the transition breadth offers significant additional information.

The mean Curie temperature $\bar{T}_{C}$ exhibits a linear dependence on the change in lattice parameter, but with two different slopes. One slope is characteristic of samples milled in the time period during which both grain size and lattice parameter decrease, and the second, larger slope is found in samples made over the range where there is increasing disorder, but the grain size remains constant. Samples that have been milled and then annealed obey the same general dependence of $\bar{T}_{C}$ on lattice parameter. In contrast, the distribution breadth $\Delta T_{C}$ is very different in milled and milled/annealed samples, indicating that while $\bar{T}_{c}$ is determined by lattice parameter, the details of local disorder are reflected by the range of temperatures over which different parts of the sample order.

The combination of mechanical milling and annealing provides a good way to separate effects due to lattice parameter changes from those due to chemical disorder. Additional study is needed, especially in the regime where the primary role of milling is grain refinement, to determine the extent of the difference in the behaviors; however, the present study confirms that $\mathrm{GdAl}_{2}$ is an excellent model system in which to study further the effects of disorder in magnetic alloys.

\section{ACKNOWLEDGMENTS}

We thank R. Lemoine and D. Schmitter for experimental assistance at UNL and S. Shastri at the Advanced Photon Source. We also thank D. Haskel, A.F. Hebard, S. Jaswal, and J.L. Shield for helpful discussions. This research was funded by the National Science Foundation through DMR9875425 and DMR-0305653, the MRSEC program of the National Science Foundation (DMR-0213808) and the Donors of the American Chemical Society Petroleum Research Fund (PRF\#40209-B5M). A.P.S. is supported by the DOE under Contract No. W-31-109-Eng-38. 
${ }^{1}$ D. Weller, S. F. Alvarado, W. Gudat, K. Schroder, and M. Campagna, Phys. Rev. Lett. 54, 1555 (1985).

${ }^{2}$ H. Tang, D. Weller, T. G. Walker, J. C. Scott, C. Chappert, H. Hopster, A. W. Pang, D. S. Dessau, and D. P. Pappas, Phys. Rev. Lett. 71, 444 (1993).

${ }^{3}$ M. Farle, K. Baberschke, U. Stetter, A. Aspelmeier, and F. Gerhardter, Phys. Rev. B 47, 11571 (1993).

${ }^{4}$ R. Kirchmayr and C. A. Poldy, in Handbook on the Physics and Chemistry of Rare Earths, edited by J. K. A. Gschneider and L. Eyring (North-Holland, Amsterdam, 1979), Vol. 1, p. 55.

${ }^{5}$ E. M. Levin, V. K. Pecharsky, and K. A. Gschneidner, J. Appl. Phys. 90, 6255 (2001).

${ }^{6}$ A. P. Malozemoff and J. P. Jamet, Phys. Rev. Lett. 39, 1293 (1977).

${ }^{7}$ B. Barbara, A. P. Malozemoff, and Y. Imry, Phys. Rev. Lett. 47, 1852 (1981).

${ }^{8}$ G. F. Zhou and H. Bakker, Phys. Rev. Lett. 73, 344 (1994).

${ }^{9}$ G. F. Zhou and H. Bakker, Phys. Rev. B 52, 9437 (1995).

${ }^{10}$ D. L. Leslie-Pelecky, E. M. Kirkpatrick, T. Pekarek, R. L. Schalek, P. Shand, D. S. Williams, and L. Yue, Mater. Res. Soc. Symp. Proc. 674, U5.1 (2001).

${ }^{11}$ D. Williams, P. M. Shand, C. Stark, T. Pekarek, R. Brown, L. Yue, and D. L. Leslie-Pelecky, J. Appl. Phys. 93, 6525 (2003).

${ }^{12}$ L. Klein, Phys. Rev. Lett. 74, 618 (1995).

${ }^{13}$ G. F. Zhou and H. Bakker, Phys. Rev. Lett. 74, 619 (1995).

${ }^{14}$ V. Petkov, J. Appl. Crystallogr. 22, 387 (1989).

${ }^{15}$ A. Gangulee, J. Appl. Crystallogr. 7, 434 (1974).

${ }^{16}$ P. Ganesan, H. K. Kuo, A. Saavedra, and R. J. DeAngelis, J. Catal. 52, 310 (1978).

${ }^{17}$ T. Proffen, and S. J. L. Billinge, J. Appl. Crystallogr. 32, 572 (1999).

${ }^{18}$ T. Mizoguchi, T. R. McGuire, S. Kirkpatrick, and R. J. Gambino, Phys. Rev. Lett. 38, 89 (1977).
${ }^{19}$ M. Bauer, M. S. S. Brooks, and E. Dormann, Phys. Rev. B 48, 1014 (1993).

${ }^{20}$ D. S. Williams, P. M. Shand, T. M. Pekarek, R. Skomski, V. Petkov, and D. L. Leslie-Pelecky, Phys. Rev. B 68, 214404 (2003).

${ }^{21}$ M. Farle, and W. A. Lewis, J. Appl. Phys. 75, 5604 (1994).

${ }^{22}$ A. Berger, G. Campillo, P. Vivas, J. E. Pearson, S. D. Bader, E. Baca, and P. Prieto, J. Appl. Phys. 91, 8393 (2002).

${ }^{23}$ G. F. Zhou, H. Yang, and H. Bakker, Mater. Sci. Forum 225, 649 (1996).

${ }^{24}$ G. F. Zhou and H. Bakker, Mater. Sci. Forum 225, 655 (1996).

${ }^{25}$ J. Bakker, I. W. Modder, G. F. Zhou, and H. Yang, Mater. Sci. Forum 235-238, 477 (1997).

${ }^{26}$ I. W. Modder, H. Bakker, and G. F. Zhou, Physica B 262, 141 (1999).

${ }^{27}$ E. Dormann, J. Magn. Magn. Mater. 6, 87 (1977).

${ }^{28}$ E. Dormann and M. Huck, Z. Phys. B 27, 141 (1977).

${ }^{29}$ E. Dormann and K. H. J. Buschow, Phys. Status Solidi B 59, 411 (1973).

${ }^{30}$ S. Jaakkola, Phys. Lett. 50A, 35 (1974).

${ }^{31}$ S. M. Jaakkola and M. K. Hänninen, Solid State Commun. 36, 275 (1980).

${ }^{32}$ W. Bao, S. Raymond, S. M. Shapiro, K. Motoya, B. Fak, and R. W. Erwin, Phys. Rev. Lett. 82, 4711 (1999).

${ }^{33}$ R. Skomski, J. Phys.: Condens. Matter 15, R841 (2003).

${ }^{34}$ D. C. Mattis, The Theory of Magnetism. I, Statics and Dynamics (Springer-Verlag, Berlin, 1988).

${ }^{35}$ M. Magnitskaya, G. Chelkowska, G. Borstel, M. Neumann, and H. Ufer, Phys. Rev. B 49, 1113 (1994).

${ }^{36}$ S. Mukherjee, R. Ranganathan, P. S. Anilkumar, and P. A. Joy, Phys. Rev. B 54, 9267 (1996).

${ }^{37}$ S. F. Fischer, S. N. Kaul, and H. Kronmüller, Phys. Rev. B 65 , 064443 (2002). 